\title{
Review
}

Shneh Sethi* and Trinad Chakraborty

\section{Molecular (real-time reverse transcription polymerase chain reaction) diagnosis of SARS-CoV-2 infections: complexity and challenges}

https://doi.org/10.1515/labmed-2020-0135

Received November 18, 2020; accepted February 8, 2021;

published online March 3, 2021

\begin{abstract}
The outbreak of coronavirus disease 2019 (COVID-19) caused by severe acute respiratory syndrome coronavirus 2 (SARS-CoV-2) was first recorded in Wuhan, China. The World Health Organization initially classified COVID-19 as a public health emergency and subsequently declared the disease a global pandemic. COVID-19 can take at least three distinct forms: severe acute distress syndrome with a potentially fatal outcome, mild respiratory illness (pneumonia with eventual recovery) and asymptomatic infection. All three disease forms have the potential to transmit the infection to healthy contacts. At present, realtime reverse transcription polymerase chain reaction (RT-PCR) is the only available laboratory tool to confirm the presence of viral RNA in patient specimens. These assays are designed to detect one or more (at least 2) SARS-CoV-2 RNA gene targets allowing the detection of the virus. Commercially available RT-PCR assays employ various gene targets of the viral genome in their assay systems. Additionally, there are differences in primer selection for the same gene region of SARS-CoV-2. At present, it is unclear whether the results from different RT-PCR assays are comparable in detecting the spectrum of COVID-19 manifestations. The purpose of the present article is twofold: first, to briefly focus on the findings of these reports; and second, to emphasize the various challenges and flaws that can potentially impact the diagnostic accuracy of RT-PCR testing for SARS-CoV-2.
\end{abstract}

\footnotetext{
*Corresponding author: Shneh Sethi, Central Institute for Clinical Chemistry and Laboratory Medicine, Klinikum Stuttgart, Kriegsbergstr. 62, 70174 Stuttgart, Germany,

E-mail: s.sethi@klinikum-stuttgart.de

Trinad Chakraborty, Institute of Medical Microbiology, German Center for Infection Research (DZIF), Partner Site Giessen-Marburg-Langen, Justus Liebig University Giessen, Giessen, Germany
}

Keywords: analytical sensititivy; clinical utility; genomic targets; real-time RT-PCR; sample type; SARS-CoV-2.

\section{Introduction}

Severe acute respiratory syndrome 2 (SARS-CoV-2) is the etiologic agent for the worldwide spread of the highly contagious coronavirus disease 2019 (COVID-19) [1, 2]. Coronaviruses (CoVs) are enveloped positive-sense singlestranded RNA viruses belonging to the Coronaviridae family. These viruses are known for their zoonotic mode of transmission to humans and respiratory transmission (via coughed/sneezed droplets/aerosols containing virus particles) from person to person [3-5].

Coronaviruses have long been recognized as the cause of seasonal cold with mild respiratory tract symptoms $[4,5]$. SARS-CoV-2 is the seventh human CoV to be identified as the causative agent of respiratory illness. Since the 1960s, four other human pathogenic CoVs designed $\mathrm{HCoV}-229 \mathrm{E}, \mathrm{HCoV}-\mathrm{NC} 63$, HCoV-OC43, and HCoV-HKU1 have been identified as the cause of mild respiratory disease among children and adults [1, 4]. During 2002-2003, a $\mathrm{CoV}$ (named SARS-CoV-1) caused an outbreak of severe respiratory infections with high mortality rates $[1,3,6]$. In 2012, another zoonotic CoV was identified as the cause of the Middle East Respiratory Syndrome (MERS) epidemic and named MERS-CoV [7, 8]. The causative agent of the 2019 pandemic, SARS-CoV-2, has shown a high degree of genomic similarity with SARS-CoV-1 [9].

The spectrum of COVID-19 has shown unique clinical features and is manifested in at least three distinct forms: severe acute respiratory distress syndrome with potential fatal outcomes; mild respiratory illness (pneumonia) with often eventual recovery; and asymptomatic infections $[10,11]$.

A differential diagnosis of COVID-19 based on clinical symptoms alone is not reliable because they are compatible 
with a spectrum of other respiratory viral infections and bacterial pneumonia. SARS-CoV-2 can be isolated by growth in cell cultures, but because of practical limitations (including biosafety), this is not an option for rapid laboratory diagnosis.

Real-time reverse transcription polymerase chain reaction (RT-PCR) detection of SARS-CoV-2-specific ribonucleic acid (RNA) in respiratory specimens from infected patients has been recommended for confirming the laboratory diagnosis of COVID-19. The development of RT-PCR assays for SARS-CoV-2 has evolved at a rapid pace, and a wide range of commercial assay kits have been developed.

There are several studies across the literature that report a variety of inconsistencies/uncertainties, which result in inaccurate laboratory diagnosis of SARS-CoV-2 infections with RT-PCR assays. The purpose of this article is twofold: first, to briefly focus on the findings of these reports; and second, to emphasize the various challenges and limitations that can potentially impact the diagnostic accuracy of RT-PCR testing for SARS-CoV-2.

\section{Detection of SARS-CoV-2 using RT-PCR assays in respiratory specimens}

Appropriate selection of the body location and the choice of specimen type(s) are critical for the detection of respiratory infections by molecular (RT-PCR) assays. Similar to other previously known coronavirus infections, SARS-CoV-2 infections primarily involve the respiratory system.

Initially, the World Health Organization (WHO) recommended the use of upper respiratory tract (URT) and/or (if necessary) lower respiratory (LRT) specimens from suspected COVID-19 patients for screening and surveillance of SARS-CoV-2 [12]. Use of URT specimens such as nasopharyngeal (NP) and oropharyngeal (OP) swabs (the term "oropharyngeal swab" is also known as throat swab") are preferred specimen types for screening purposes because they are easy to collect and process for RT-PCR. LRT specimens such as sputum are also easy to collect (if produced) but require a preprocessing step for RNA extraction. Other LRT specimens, such as bronchoalveolar lavage (BAL) and airway aspirates require invasive procedures and thus are not preferred specimen types for screening purposes. LRT specimens are recommended in suspected COVID-19 cases with severe progressive respiratory disease.

Several studies have focused on the performance of URT and LRT specimen types, time of collection (post disease onset), viral loads (the term viral load has been used in the text to indicate the amount of SARS-CoV-2 RNA material [copies/mL, genome equivalents, Cycle threshold (Ct) values] in the test samples; it may not necessarily be equated with the number of infectious particles [virion]) and disease detection rates with inconsistent and conflicting results.

\section{Nasopharyngeal vs. oropharyngeal swab samples}

A study involving symptomatic patients $(\mathrm{n}=18)$ compared viral loads (soon after disease onset) in a total of 72 sequential specimens of nasal swabs (NP and midturbinate) and 72 throat swabs. Quantitative real-time polymerase chain reaction (RT-qPCR) testing for SARS-CoV-2 RNA showed higher viral loads in NP than in throat specimens. A similar trend was observed in a single asymptomatic patient who was included in the study [13]. A cluster of five cases with a clinical diagnosis of probable COVID-19 showed high viral loads in NP swabs within $24 \mathrm{~h}$ of illness. In four cases, viral loads declined (but remained detectable) over time and tested negative between illness days four to nine. In a critical patient who died on day 14 of illness, persistent SARS-CoV-2 RNA was detected in NP swabs, BAL fluid and pleural fluid [14]. An investigation of serial NP and OP specimens from a cluster of hospitalized patients $(\mathrm{n}=10)$ with mild COVID-19 showed high viral loads in both NP and OP swabs during days one to five of illness and then declined after day five.

The authors argued that high viral loads and positive virus isolation from early swab samples (16.66\%) indicate viral replication in URT tissues [15]. Multiple specimen types from patients $(\mathrm{n}=205)$ with suspected COVID-19 were analyzed for the presence of viral RNA. Pharyngeal swabs were collected in most patients one to three days after hospitalization (pre-date time of collection after development of symptoms is not provided). Higher positivity was observed in NP swabs (62\%, five of eight) than in OP swabs (32\%, 126 of 398) [16].

Examination of 12 hospitalized patients with confirmed COVID-19 revealed higher viral loads in the URT specimens during the first week of illness. A total of 408 samples were tested for the presence of viral RNA. The results showed RT-PCR positivity in 11 of 12 OP swabs, 12 of $12 \mathrm{NP}$ swabs and 6 of 6 sputum specimens. Notably, viral RNA was detectable in 7 cases after the disappearance of symptoms, i.e., longer than two to three weeks after hospitalization [17]. A separate study concluded that $50 \%$ of OP swabs tested positive for SARS-CoV-2 RNA at first 
testing, whereas after day five, only $25 \%$ of OP swabs were recorded as positive [18]. Comparative assessment of viral loads (mild vs. severe cases) was conducted in 76 patients. NP swabs from 46 (61\%) mild cases and 30 (39\%) severe cases revealed higher viral loads in severe cases than in mild cases [19].

The evidence from the abovementioned studies indicates that SARS-CoV-2 has the potential to colonize and/ or infect URT and LRT tissues. Thus, viral RNA is detectable by RT-PCR assays in the NP and OP swabs of COVID-19 patients; viral loads are higher in NP swabs than in OP swabs. Overall, the data suggest that the SARS-CoV-2 detection yield is higher in sputum samples, BAL and other respiratory aspirates, which is consistent with the higher viral loads observed in LRT vs. URT specimens.

\section{Upper respiratory tract vs. lower respiratory tract samples}

Only a few studies comparing the performance of URT vs. LRT for detecting SARS-CoV-2 RNA have been reported.

RT-PCR testing of paired specimens of sputum and oropharyngeal swabs from 52 COVID-19 cases revealed higher positivity rates of SARS-CoV-2 RNA in sputum (76.9\%) compared to $44.2 \%$ in OP swabs [20]. In a study on SARS-CoV2 RNA detection in multiple clinical specimens, BAL fluid showed the highest positive rates (93\%), followed by sputum (72\%), nasal swabs (63\%) and OP swabs (32\%) [16].

Paired swabs (NP and OP) and sputum specimens were collected from 7 hospitalized patients with mild illness on days two and four after disease onset and tested for the presence of SARS-CoV-2 RNA. In two cases, swab samples had higher viral loads, while the other two cases showed the reverse. In three other cases, similar viral loads were detected in both swab and sputum samples [15]. A comparative assessment of viral loads in throat swabs and sputum specimens showed higher SARS-CoV-2 RNA levels than those detected in throat swabs [21]. A report [22] described the detection of SARS-CoV-2 RNA in saliva specimens collected from COVID-19 patients. More data are needed to ascertain the use of this specimen type for the diagnosis of COVID-19.

\section{False negative RT-PCR testing of upper respiratory tract samples: impact of repeat tests}

There are reports of "false-negative" findings on swab specimens (NP and/or OP) in patients where clinical features and computer tomography (CT) scan abnormalities are compatible with those of COVID-19.

Three cases of suspected COVID-19 pneumonia with abnormal CT imaging were negative for SARS-CoV-2 RNA on initial testing of NP swabs. However, after repeated RT-PCR testing, these results became positive [23]. Reportedly, five out of 167 patients with clinical features and CT scan abnormalities compatible with COVID-19 tested negative for viral RNA in throat swabs; repeated swab testing yielded positive results [24]. Testing of URT specimens for SARS-CoV-2 RNA in pharyngeal swabs showed high "false negative" rates. Among 610 hospitalized patients, 168 cases were positive on initial testing, and 384 (63.0\%) were negative. On second testing, 48 cases (12.5\%) tested positive, 27 (7.0\%) tested dubiously positive and $280(19.8 \%)$ tested negative [25]. Two separate reports $[26,27]$ have described solitary cases of suspected COVID-19 where clinical findings and CT scan features resembled COVID-19 pneumonia, but repeated (at least two) testing of NP or OP swabs showed negative results for viral RNA. Subsequent tests of the BAL fluid in one case [27] and sputum in the second case [26] showed positive results.

There are case reports describing COVID-19 patients who were clinically cured and tested negative for viral RNA in repeated NP and/or OP swabs and were then discharged. However, follow-up tests were positive in sputum samples [28, 29].

A recent study presented large data related to the detection of SARS-CoV-2 RNA in NP swab samples [30]. The findings of this study exemplify and corroborate the abovementioned uncertainties of RT-PCR testing on URT swab samples (for details, refer to the clinical utility Section 3.3).

The aforementioned evidence raises several concerns related to the timely and reliable diagnosis of COVID-19 based on URT swabs. The time assessment of SARS-CoV-2 RNA detection depends not on single testing, but successive multiple tests are required, which impacts the timely laboratory diagnosis of the disease. The issues of frequent conversion of results (negative to positive or vice versa) may be ascribed to the complex interactions between SARS-CoV-2 virulence determinants and host innate immune defense barriers at the initial site of viral entry and shedding, which is believed to be the nose, and probably the oral cavities. Little is known about the underlying events after SARS-CoV-2 entry into the host, which promote virus colonization (asymptomatic carriers) and those that limit (resist) or enhance virus replication (low vs. high viral loads) and shedding. It is likely that the initial viral exposure dose (low or high inoculum) plays a critical role in the outcome of these interactions. Viral load analysis studies 
have shown that there is a short time frame (few days; often one to five days) after symptoms begin when viral loads peak in URT samples. Thus, NP or OP swabs collected late in the disease process may test negative for SARS-CoV-2 RNA.

It has been noted that there are instances where repeat testing is initially positive, then negative and, upon retesting, positive again. This is probably a reflection of intermittent virus shedding within the pharyngeal region of the URT. An alternative possibility (but not proven) is that virus particles (droplets/aerosols) originating from the LRT tissues are seeded in the pharyngeal sites of the URT.

It is also conceivable that in some cases, the presence of low viral loads is undetectable by RT-PCR assays, which results in false negative findings and subsequently converts positive results to repeat tests. It must be emphasized that the detection of viral RNA in samples from "cured" patients or those in convalescence does not necessarily imply that viable or infectious viruses have been detected. The observed positivity in such cases may be due to the persistence of low levels of residual RNA.

Collectively, all the above mentioned factors may contribute to the reported false-negative results and the inaccuracies of the RT-PCR tests on clinical URT samples.

\section{Suitability of sputum samples for detection of SARS-CoV-2 RNA}

Available evidence (refer Section 2.2) suggests that because of the consistency and presence of high viral loads, sputum is better suited for the detection of SARS-CoV-2 RNA than URT swab samples. Sputum samples are easy to collect and are preferred specimen types for the molecular diagnosis of several respiratory infections. For COVID-19 patients who may not be able to produce sputum, induced sputum (IS) samples that are safe to induce (do not require invasive procedures) are commonly used [31]. From the biosafety aspects, hospital personal collecting sputum samples are exposed to the same risk level as for collecting NP swabs. However, sputum, because of its high viscosity (varies from sample to sample), needs a preprocessing homogenization step for the extraction of intact viral RNA.

At present, a standardized and validated protocol for this step is not available [20]. PCR testing laboratories need to develop and validate their own protocols for this crucial step, which may affect the final overall sensitivity of the PCR assays. Additionally, current assays and PCR testing platforms for large volume testing are designed and validated for swab-based testing. In view of these technical obstacles, sputum samples are not an acceptable choice for large volume screening/surveillance purposes. However, sputum or IS samples are better suited for testing patients with a mild form of COVID-19 and asymptomatic "carriers" of SARS-CoV-2. The use of sputum or IS for the detection of SARS-CoV-2 RNA may reduce the false-negative rate of RT-PCR-based testing.

\section{RT-PCR assays for detection of SARS-CoV-2 RNA}

In addition, considering the choice of specimen type and its body location as described above, other preanalytical steps can affect the performance of the assays [32, 33]. Briefly, the proper specimen collection procedure, timing of collection, storage and transport to the laboratory are critical for the success of molecular assays. Additionally, improper processing of the specimen for RNA extraction (manual/instrument) will impact the overall sensitivity of the assay. It is essential that RNA is present in the test sample in undegraded state and detectable amounts.

\section{Genomic targets}

A challenge for the development of RT-PCR assays for diagnostic use is the selection of appropriate targets in the SARS-CoV-2 genome and the design of reliable primer sequences for detecting the target gene(s). During the early phase of the COVID-19 pandemic, the WHO issued guidelines for developing RT-PCR assays to diagnose COVID-19 [12]. The preferred viral RNA gene targets recommended included nucleocapsid $(N)$, spike $(S)$, envelope $(E)$ and RNA-dependent-RNA-polymerase $(R d R p)$. It was suggested to employ at least two different gene targets, one for screening CoVs related to SARS-CoV-2 and the other for confirmation of COVID-19 virus. Initially, 2 RT-PCR protocols for the diagnosis of COVID-19 were endorsed by the WHO [34]. The protocol developed at the Institute of Virology, Charité Hospital, Berlin, Germany, uses two gene targets of SARS-CoV-2: the Sarbeco $E$ gene for screening bat-related CoVs and the $R d R p$ gene with two primer/probe sets, one for batrelated CoVs and the other for confirmation of SARS-CoV-2 [35]. The second assay protocol developed by the Centers for Disease Control, USA, employs three primer/probe sets to detect three regions $(N 1, N 2$, and $N 3)$ of the SARS-CoV-2 N gene [36].

SARS-CoV-2 belongs to the beta group within the Sarbecovirus subgenus of CoVs. A typical coronavirus RNA 
genome is composed of two untranslated (UTR) regions located between the $5^{\prime}$ and $3^{\prime}$-poly(A) ends of the genome. The coding genes are located between the two UTRs in the following order: $5^{\prime}$ replicase complex of the open reading frame (ORF/1ab), $S$ gene, $E$ gene, $M$ gene, $N$ gene and 3 '-poly(A) tail. SARS-CoV-2 contains several ORFs; the replicase ORF1/ab gene consists of two ORFs (ORF1a and ORF1b). The $O R F$ gene encodes a number of nonstructural proteins (nsps); nsp 12 and nsp 13 encode the RdRp and helicase (Hel) proteins, respectively. The $3^{\prime}$ terminal region of the genome is involved in encoding structural proteins (S, E, M, and $\mathrm{N}$ ) and a number of accessory proteins [37-39].

The availability of the full genome sequence of SARS-CoV-2 RNA facilitated the development of PCR-based assays for the diagnosis of COVID-19. Several in-house assay protocols have been developed by independent research/health institutions. A variety of RT-PCR assay kits for detecting SARS-CoV-2 RNA are available commercially (listed elsewhere: [32, 40]). The preferred gene targets include $E, N, S, O R F / 1 a b$ and $R d R p$. A recent report showed the high sensitivity and specificity of an RT-PCR assay targeting the RdRp/Hel region of SARS-CoV-2 RNA [41].

\section{Analytical validity}

While multiple RT-PCR assay kits have been developed, a comparative assessment of the SARS-CoV-2 detection performance of these assays is difficult. Unfortunately, the current assays available are not identical; they employ not only different targets of SARS-CoV-2 RNA in their assay system but also variability in the design of primers/probe sets used for detecting different regions of the same locus [42]. There are also important differences in the operational design of the PCR testing instruments (amplification conditions, extraction of RNA and essential reagents). Importantly, there are no clear guidelines or certified standard material with exact levels of SARS-CoV-2 for the validation of different RT-PCR assays. Three independent studies [43-45] compared (RT-qPCR) the performance of different primer/probe sets used in SARS-CoV-2 detection RT-PCR assays posted by the WHO on their homepage. The studies employed identical operational PCR conditions and reagents for the evaluation. The primer/probe sets included were those developed by the Centers for Disease Control, USA (USCDC), Centers for Disease Control, China (CCDC) Charité Hospital Institute of Virology, Berlin, Germany (Charité), Hong Kong University (HKU) and University of Washington, USA (USUW). The assays targeted the ORF1/ $a b, O R F 1 b$-nsp14, RdRp, $E$ and $N$ genes of SARS-CoV-2. The first study [43] assessed the performance of 7 primers/ probe sets by using panels of NP and/or OP swabs not tested for COVID-19 ( $\mathrm{n}=\mathrm{ca}$. 300), samples positive for different viruses $(n=18)$, samples positive for non-CoV-2 CoVs $(n=25)$, samples from confirmed COVID-19 patients $(\mathrm{n}=10)$ and 22 samples negative for SARS-CoV-2 RNA. A positive standard sample with $3.16 \times 10^{5}$ equivalents of the viral genome was used to estimate the lower limit of detection (LOD).

Analysis of USCDC N1, N2, and N3 sets and RdRp-SARS and Sarbeco $E$ sets (Charité) using 10 positive COVID-19 positive samples showed that all the sets, except the USCDC N3 set, identified the positive samples. The CDC has removed its $N 3$ primer/probe set from the CDC-US assay protocol (http://www-360dx com/per/cdc-revises-sarsCoV-2 assay protocol-surveillance-testing-track-startnext-week.) It was concluded that the most sensitive primer/probe sets were the $E$ gene (Charité) and USCDC N1 and N2, which detected approximately 6.3 genomic equivalents per reaction; the least sensitive was the $R d R p$ set (Charité). None of the assays showed evidence of cross reactivity with other respiratory viruses. In the second study, the availability of pre-COVID-19 NP swab specimens (year 2017), viral transcript standards, isolated full-length SARS-CoV-2 RNA and clinical samples from COVID-19 patients assisted the comparative evaluation of primer/probe sets. The sensitivities of different sets varied from 63 to $75 \%$ : the Sarbecco E (Charité) and ORF1 (HKU) primer sets were the most sensitive (75\%), each detecting NP swab samples spiked with 10 viral genome equivalents $/ \mu \mathrm{L}$. At $10^{2}$ virus genome equivalents, all sets, except the RdRp-SARS set (Charité), could detect COVID-19 virus and differentiate between negative samples. The RdRp-SARS set tested negative for all $10^{0}-10^{2}$ genome equivalent concentrations. It was inferred that the low sensitivity of the RdRp-SARS set may be the result of a mismatch in the reverse primer and not due to any sequence variations. The $C C D C-N$, CDCC-ORF-1, USCDC-N2 and USCDC-N3 sets showed nonspecific background amplification with SARS-CoV2-negative NP samples. The authors suggested that this may have resulted from cross-reactions with seasonal CoVs circulating in 2017.

The third study employed reversely transcribed cDNAs from pooled RNAs extracted from NP swabs of healthy individuals (mock negative) or COVID-19 patients (mock positive) to compare the performance of eight different primer/ probe sets [45]. RT-qPCR assays were performed simultaneously according to the protocols of the institutions who designed the sets. Amplification of nonspecific products was observed in mock negative controls (false positive) with $U S C D C-N 1$ and $N 2$ and $C C D C-N$. The sets with no background 
signals in mock negatives (USCDC-N3, HKU-N, Charité E and $H K U$-ORF) failed to identify all $10^{-4}$ diluted positive samples. The most sensitive set was CCDC-ORF, which detected $3 / 10$ positive samples. It was inferred that all eight primer/probe sets could correctly detect $10^{-2}$ - and $10^{-1}$-diluted positive samples, but none could correctly identify all $10^{-4}$-diluted positives.

The findings of these three studies suggest that the primer/probe sets employed in commonly used RT-PCR assays show significant variability in their sensitivities in detecting low-viral load samples. Current RT-PCR assays used for identifying patients with COVID-19 have high analytical sensitivities and specificities, as declared in their test instruction sheets. Most of the assays may detect clinical specimens with high viral loads, but their performance in identifying low viral load samples is limited.

\section{Clinical utility}

As a result of the emergency situation created by the COVID-19 outbreak, regulatory authorities were unable to assess the clinical validity and reliability of the assays. A review article dealing with the laboratory diagnostic aspects of COVID-19 concluded that the positive results with the current assays account for approximately $50 \%$ of the clinically confirmed assays [32].

In the absence of a true "gold standard", the accurate sensitivity and specificity of current RT-PCR assays for detecting SARS-CoV-2 RNA is difficult to predict. Nevertheless, contrasting approaches have been employed to ascertain the clinical utility of the assays for COVID-19 diagnosis.

A very recent study enrolled 3,432 hospitalized patients based on their medical records for repeat SARS-CoV2 RNA testing. Of these, 2,630 patients initially tested negative, and 880 were positive for viral RNA in their NP or OP swabs [30]. Based on the results of repeat testing, the clinical sensitivity of RT-PCR assays was estimated to be between 58 and $96 \%$ depending upon the unknown number of false-negative results in single tested patients. The results also revealed various patterns of conversions and reconversions. Forty-eight cases were identified that initially tested positive, then converted negative and then positive again. The authors estimated that the conversions of initial positive to negative occur between 15 and 20 days with $50 \%$ conversions noted after 28 days. The probability (25\%) of conversions among initially negatives to positives was 20 days. Fluctuations of results between positive and negative results were observed among a significant number of patients. It was suggested that repeat tests be performed among highly suspected patients between 15 and
20 days after a positive test and within $24-48 \mathrm{~h}$ after a negative test. These estimates are based on the results of patients with a severe form of COVID-19 or with high suspicion of the disease. The estimates among cases with mild COVID-19 and those with asymptomatic infections may be different.

A related retrospective study used the results of multiple RT-PCR tests and CT chest scans to analyze the conversions of RT-PCR results in relation to CT scans. The study used the medical records of 1,014 patients from Wuhan (China), of whom 601 had positive RT-PCR results, 413 tested negative for viral RNA and 888 had positive CT scans. The analysis revealed that of the 601 RT-PCR-positive cases, $308(75 \%)$ had positive CT scans. Based on RT-PCR as the reference standard, the sensitivity of chest CT scans in diagnosing COVID-19 was estimated to be approximately $97 \%$ (580 of 601 cases), with a specificity of $25 \%$ (105 of 413 cases) and an accuracy of 68\% (685 of 1,014 cases). Notably, $81 \%$ of the patients who tested negative for SARS-CoV-2 RNA and had positive CT scans were subsequently reclassified as highly likely cases of COVID-19 [46].

The uncertainties of the results with these assays (single/ multiple tests) impact the timely diagnosis of COVID-19. This is of concern for patient management, prevalence of disease transmission (quarantine and discharge) and importantly for the patient himself. Thus, it is important that the "gold standard" criteria for detecting COVID-19 include, in addition to RT-PCR testing, additional disease-related criteria such as conventional lung scan features and serological testing results for specific antibodies, especially IgM for establishing early diagnosis. The diagnosis of "asymptomatic carriers" remains a challenge. The use of immunological "biomarkers" such as the secretion of proinflammatory cytokines may assist in distinguishing "asymptomatic carriers" from presymptomatic carriers".

\section{Concluding remarks}

This article is based on the findings of selected reports from the current literature related to the molecular diagnostic aspects of COVID-19. It summarizes data on the reported failures of RT-PCR tests on URT swab samples and the factors that may account for them. It is concluded that current assays based on URT specimens are not suitable for diagnosing COVID-19 in its different phases due to its complex biological nature. Despite the flaws observed in the testing of URT samples, they remain a preferred sample type for high-volume screening and surveillance purposes. Sputum (if produced) or IS samples represent a better sample type for the rapid, consistent and reliable detection of SARS-CoV-2 
RNA in hospitalized cases of mild COVID-19. As this article is being written, we are in the middle of a COVID-19 pandemic. A large proportion of the world population has been infected with the virus. Presumably, the number of infected individuals in the general population is higher than that identified so far and has not yet been tested. These infected individuals are a potential source of the onward spread of the virus. Intensive efforts are underway for the detection of infected individuals through large volume screening surveillance using RT-PCR assays to prevent or minimize the spread of COVID-19. SARS-CoV-2 is highly contagious, and to prevent its transmission to healthy individuals, it is urgent to obtain results rapidly. This is not feasible with RT-PCR assays, as it takes time for transport and testing the specimens in special laboratories (ca. 24-72 h).

In contrast, (lateral flow) SARS-CoV-2-specific antigen detection tests, which are now available commercially, provide the results rapidly (ca. $15 \mathrm{~min}$ ) on the spot and are suitable for COVID-19 quarantine containment purposes. The drawback of antigen detection tests is that they reportedly fail to detect both presymptomatic (initiation phase) and asymptomatic infected individuals. Finally, optimization and harmonization of current RT-PCR assays is needed with respect to the selection of appropriate targeted genes of SARS-CoV-2 as well as corresponding high sensitivity primers/probe sets; this may increase the detection rate of low viral load specimens. The rapid release of genome sequences of SARS-CoV-2 was important for the development of rapid, relatively inexpensive and sensitive RT-qPCR assays during the onset of the pandemic. As SARS-CoV-2 continues to acquire genetic changes, accelerated determination of genome sequences provides the tool vital to monitor and validate the expected sensitivity of various diagnostic assays even within a locality. They, in particular, enable the development of newer assays relevant for the investigation of spatiotemporal spread of viruses and their transmissibility. Moreover, in a clinical context, it is important to standardize the methodology for estimating PCR cycle threshold values (Ct values) in RT-qPCR assays which indicate amounts of viral loads in URT samples. The determination of viral loads is used for monitoring disease severity and response to therapeutic interventions. The use of URT swab suspensions is not suitable for quantitation purposes. Swab suspensions are prone to errors attributable to sample collection and mucosal surface cell mass which underlines that a standardized method is needed. Ct values from realtime RT-PCR assays which are not quantitative (lack of normalization against internal controls) however, allow to safely determine SARS-CoV-2 infected patients from noninfected individuals.

Research funding: None declared.

Author contributions: All authors have accepted responsibility for the entire content of this manuscript and approved its submission.

Competing interests: Authors state no conflict of interest.

\section{References}

1. Coronaviridae Study Group of the International Committee on Taxonomy of Viruses. The species Severe acute respiratory syndrome-related coronavirus: classifying 2019-nCoV and naming it SARS-CoV-2. Nat Microbiol 2020;5: 536-44.

2. Del Rio C, Malani PN. COVID-19-New insigths on a rapidly changing epidemic. JAMA 2020;323:1339-40.

3. Zumla A, Chan JF, Azhar E, Hui DS, Yuen KY. Coronaviruses - drug discovery and therpeuticoptions. Nat Rev Drug Discov 2016;15: 327-47.

4. Cui J, Li F, Shi ZL. Origin and evolution of pathogenic coronaviruses. Nat Rev Microbiol 2019;17:181-92.

5. Wang C, Horby PW, Hayden FG, Gao GF. A novel coronavirus outbreak of global health concern. Lancet 2020;395:470-3.

6. Kuiken T, Fouchier RA, Schutten M, Rimmelzwaan GF, van Amerongen G, van Riel D, et al. Newly discovered coronavirus as the primary cause of severe acute respiratory syndrome. Lancet 2003;362:263-70.

7. Zaki AM, van Boheemen S, Bestebroer TM, Osterhaus AD, Fouchier RA. Isolation of a novel coronavirus from a man with pneumonia in Saudi Arabia. N Engl J Med 2012;367:1814-20.

8. Zumla A, Hui DS, Perman S. Middle East respiratory syndrome. Lancet 2015;386:995-1007.

9. Zhou P, Yang XL, Wang XG, Hu B, Zhang L, Zhang W, et al. A pneumonia outbreak associated with a new coronavirus of probable bat origin. Nature 2020;578:270-3.

10. Huang C, Wang Y, Li X, Ren L, Zhao J, Hu Y, et al. Clinical features of patients infected with 2019 novel coronavirus in Wuhan, China. Lancet 2020;395:497-506.

11. Zhou X, Li Y, Li T, Zhang W. Follow-up of asymptomatic patients with SARS-CoV-2 infection. Clin Microbiol Infect 2020;26:957-9.

12. WHO. Laboratory testing for coronavirus disease 2019 (COVID-19) in suspected human cases: interim guidance, 19 March, 2020. Geneva: WHO; 2020.

13. Zou L, Ruan F, Huang M, Liang L, Huang H, Hong Z, et al. SARS-CoV-2 viral load in upper respiratory specimens of infected patients. N Engl J Med 2020;382:1177-9.

14. Lescure FX, Bouadma L, Nguyen D, Parisey M, Wicky PH, Behillil S, et al. Clinical and virological data of the first cases of COVID-19 in Europe: a case series. Lancet Infect Dis 2020;20: 697-706.

15. Wolfel R, Corman VM, Gugemos W, Seilmaier M, Zang S, Muller MA, et al. Virological assessment of hospitalized patients with COVID-2019. Nature 2020;581:465-9. 
16. Wang W, Xu Y, Gao R, Lu R, Han K, Wu G, et al. Detection of SARS-CoV-2 in different types of clinical specimens. JAMA 2020; 323:1843-4.

17. COVID-19 Investigation Team. Clinical and virologic characteristics of the first 12 patients with coronavirus disease 2019 (COVID-19) in the United States. Nat Med 2020;26:861-8.

18. Zhang W, Du RH, Li B, Zheng XS, Yang XL, Hu B, et al. Molecular and serological investigation of 2019-nCoV infected patients: implication of multiple shedding routes. Emerg Microb Infect 2020;9:386-9.

19. Liu Y, Yan LM, Wan L, Xiang TX, Le A, Liu JM, et al. Viral dynamics in mild and severe cases of COVID-19. Lancet Infect Dis 2020;20:656-7.

20. Lin C, Xiang J, Yan M, Li H, Huang S, Shen C. Comparison of throat swabs and sputum specimens for viral nucleic acid detection in 52 cases of novel coronavirus (SARS-Cov-2) infected pneumonia (COVID-19). Clin Chem Lab Med 2020;58:1089-94.

21. Pan Y, Zhang D, Yang P, Poon LLM, Wang Q. Viral load of SARS-CoV-2 in clinical samples. Lancet Infect Dis 2020;20:411-2.

22. To KK, Tsang OT, Yip CC, Chan KH, Wu TC, Chan JM, et al. Consistent detection of 2019 novel coronavirus in saliva. Clin Infect Dis 2020;71:841-3.

23. Xu J, Wu R, Huang H, Zheng W, Ren X, Wu N, et al. Computed tomographic imaging of 3 patients with coronavirus disease 2019 pneumonia with negative virus real-time reverse-transcription polymerase chain reaction test. Clin Infect Dis 2020;71:850-2.

24. Xie X, Zhong Z, Zhao W, Zheng C, Wang F, Liu J. Chest CT for typical 2019-nCoV pneumonia: relationship to negative RT-PCR testing. Radiology 2020:200343.

25. Li Y, Yao L, Li J, Chen L, Song Y, Cai Z, et al. Stability issues of RT-PCR testing of SARS-CoV-2 for hospitalized patients clinically diagnoses with COVID-19. J Med Virol 2020;92:903-8.

26. Hornuss D, Laubner K, Monasterio C, Thimme R, Wagner D. COVID-19 associated pneumonia despite repeatedly negative PCR-analysis from oropharyngeal swabs. Dtsch Med Wochenschr 2020;145:844-9.

27. Winichakoon P, Chaiwarith R, Liwsrisakun C, Alee P, Goonna A, Limsukon A, et al. Negative nasopharyngeal and oropharyngeal swabs do not rule out COVID-19. J Clin Microbiol 2020;58.

28. Lan L, Xu D, Ye G, Xia C, Wang S, Li Y, et al. Positive RT-PCR test results in patients recovered FROM COVID-19. JAMA 2020;323: 1502-3.

29. Carmo A, Pereira-Vaz J, Mota V, Mendes A, Morais C, da Silva AC, et al. Clearance and persistence of SARS-CoV-2 RNA in patients with COVID-19. J Med Virol 2020;92:2227-31.

30. Green DA, Zucker J, Westblade LF, Whittier S, Rennert H, Velu P, et al. Clinical performance of SARS-CoV-2 molecular tests. J Clin Microbiol 2020;58:e00995-20.

31. Han H, Luo Q, Mo F, Long L, Zheng W. SARS-CoV-2 RNA more readily detected in induced sputum than in throat swabs of convalescent COVID-19 patients. Lancet Infect Dis 2020;20:655-6.

32. Yan Y, Chang L, Wang L. Laboratory testing of SARS-CoV, MERS-CoV, and SARS-CoV-2 (2019-nCoV): current status, challenges, and countermeasures. Rev Med Virol 2020;30:e2106.
33. Lippi G, Simundic AM, Plebani M. Potential preanalytical and analytical vulnerabilities in the laboratory diagnosis of coronavirus disease 2019 (COVID-19). Clin Chem Lab Med 2020; 58:1070-6.

34. WHO. Laboratory testing for coronavirus disease 2019 (COVID-19) in suspected human cases. Interim guidance 2 March, 2020. Available from: https://www.who.int/publications/i/item/ 10665-331501 [Accessed 9 Mar 2020].

35. Corman VM, Landt O, Kaiser M, Molenkamp R, Meijer A, Chu DK, et al. Detection of 2019 novel coronavirus (2019-nCoV) by realtime RT-PCR. Euro Surveill 2020:25.

36. Centers for Disease Control and Prevention-CDC2019-Novel Coronavirus (2019-nCoV). Real-time RT-PCR diagnostic panel. Available from: https=//www.fda-gov/media/134922/download [Accessed 9 Mar 2020].

37. Malik YS, Sircar S, Bhat S, Sharun K, Dhama K, Dadar M, et al. Emerging novel coronavirus (2019-nCoV)-current scenario, evolutionary perspective based on genome analysis and recent developments. Vet Q 2020;40:68-76.

38. Wu A, Peng Y, Huang B, Ding X, Wang X, Niu P, et al. Genome composition and divergence of the novel coronavirus (2019-nCoV) originating in China. Cell Host Microbe 2020;27:325-8.

39. Masters PS. Coronavirus genomic RNA packaging. Virology 2019; 537:198-207.

40. Pang J, Wang MX, Ang IYH, Tan SHX, Lewis RF, Chen Jl, et al. Potential rapid diagnostics, vaccine and therapeutics for 2019 novel coronavirus (2019-nCoV): a systematic review. J Clin Med 2020:9.

41. Chan JF, Yip CC, To KK, Tang TH, Wong SC, Leung KH, et al. Improved molecular diagnosis of COVID-19 by novel; highly sensitive and specific COVID-19-RdRp/Hel real-time reverse transcription $P C R$ assay validated in vitro with clinical specimens. J Clin Microbiol 2020;58:e00310-20.

42. John Hopkins Center for Health Security. Comparison of national RT-PCR primers, probes and protocol for SARS-CoV-2 diagnostics; 2020. Available from: https://www. centerforhealthsecurity.org/resources/COVID-19/COVID-19-factsheets/200410-RT-PCR.pdf.

43. Nalla AK, Casto AM, Huang MW, Perchetti GA, Sampoleo R, Shrestha L, et al. Comparative performance of SARS-CoV-2 detection assays using seven different primer-probe sets and one assay kit. J Clin Microbiol 2020:58.

44. Vogels CBF, Brito AF, Wyllie AL, Fauver JR, OttI M, Kalinich CC, et al. Analytical sensitivity and efficiency comparisons of SARS-CoV-2 RT-qPCR primer-probe sets. Nat Microbiol 2020;5: 1299-305.

45. Liu X, Feng J, Zhang Q, Guo D, Zhang L, Suo T, et al. Analytical comparisons of SARS-CoV-2 detection by qRT-PCR and dd PCR with multiple primers/probe sets. Emerg Microb Infect 2020;9: 1175-9.

46. Ai T, Yang Z, Hou H, Zhan C, Chen C, Lv W, et al. Correlation of Chest CT and RT-PCR testing in Coronavirus disease 2019 (COVID-19) in China. A report of 2014 cases. Radiology 2020;296:E32-40. 